\title{
Time-Limited Trials: a qualitative study exploring the role of time in decision-making on
}

the Intensive Care Unit

\section{Corresponding Author}

Name: Bradley Lonergan

Affiliations: University Hospitals of Morecambe Bay (UHMB), Lancaster University

Address: Royal Lancaster Infirmary, Ashton Road, Lancaster, UK. LA1 4RP

Email: bradleylonergan@doctors.org.uk

Tel: $+44(0) 7845711330$

ORCID: 0000-0001-8648-7048

\section{Authors}

Name: Laura Machin

Affiliations: Lancaster University (Lancaster Medical School)

Address: Lancaster University, Furness Building, Hazelrigg Lane, Lancaster, UK. LA1 4YG

Email: l.machin@lancaster.ac.uk

Tel: +44 (0)1524 594973

ORCID: 0000-0002-6717-959X

Name: Rachel Markham

Affiliations: University Hospitals of Morecambe Bay (UHMB), Lancaster University

Address: Royal Lancaster Infirmary, Ashton Road, Lancaster, UK. LA1 4RP

Email: Rachel.markham@mbht.nhs.uk

Name: Alexandra Wright

Affiliations: St Helens and Knowsley Teaching Hospitals

Address: Aintree University Hospital NHS Foundation Trust, Lower Lane, Fazakerley, Liverpool, UK. L9 7AL

Email: alwright90@doctors.org.uk

Conflicts of Interests: none to declare, no funding provided 


\section{Abstract}

Background: Withholding and withdrawing treatment are deemed ethically equivalent by most Bioethicists, but intensivists often find withdrawing more difficult in practice. This can lead to futile treatment being prolonged. Time Limited Trials (TLTs) have been proposed as a way of promoting timely treatment withdrawal whilst giving the patient the greatest chance of recovery. Despite being in UK guidelines, TLTs have been infrequently implemented on Intensive Care Unit (ICU). We will explore the role of time in ICU decision-making and provide a UK perspective on debates surrounding TLTs.

Methods: This qualitative study recruited 18 participants (9 doctors, 9 nurses) from two ICUs in North West England for in-depth, one-to-one semi-structured interviews. A thematic analysis was performed of the data.

Results: Our findings show time is utilised by ICU staff in a variety of ways including managing uncertainty when making decisions about a patient's prognosis or the reversibility of a disease; constructing relationships with patients' relatives; communicating difficult messages to patients' relatives; justifying resource allocation decisions to colleagues; and demonstrating compassion towards patients and their families.

Conclusions: Time shifts the balance towards greater certainty in ICU decision-making, by demonstrating futility, and can ease the difficult transition for staff and families from active treatment to palliation. However, this requires clear and open communication, both within the ICU team and with the family, being prioritised when time is used in decision-making.

Keywords: Intensive Care Unit; medical uncertainty; End of life care; Treatment withdrawal; ICU decision making 


\section{Background}

Treatment on the Intensive Care Unit (ICU) is only appropriate if it offers a reasonable chance of recovery to a quality of life that is acceptable to that patient ${ }^{1}$. Deciding which treatments are clinically appropriate or futile, and what level of recovery is acceptable can be difficult, particularly for non-communicative patients without capacity ${ }^{2}$. Futile treatments should be withheld from the outset or withdrawn in a timely fashion to avoid prolonging inevitable dying. 'Futility' in clinical practice has proven difficult to define, as intensivists predicting a poor outcome risk creating a self-fulfilling prophecy3. Using the term 'medically inappropriate' highlights that these decisions are value judgements made by intensivists and promotes discussion of what is appropriate, though it is no more objective or easier to explain to the family ${ }^{1}$. Intensivists can utilise clinical guidelines and scoring algorithms, such as $\mathrm{APACHE}^{4,5}$ to predict patients' mortality risk, but studies show that senior intensivists are only moderately good at predicting outcomes for individual patients and their unique circumstances ${ }^{5}$. In practice, intensivists often rely on their experience, intuition and heuristics to manage uncertainty when making decisions involving patients' prognoses and disease reversibility ${ }^{5-7}$. The pressure on intensivists to reach clinically sound decisions is magnified by financial considerations, with a night on ICU costing almost five times more than on a general ward, and compounded by the inadequate number of beds and poor staffing levels in ICUs in the United Kingdom $(\mathrm{UK})^{8,9}$.

A novel strategy for decision-making on ICU is formulating an agreement between patients, relatives and staff to use a treatment for a defined period and observing a patient's response. If the patient improves according to agreed clinical outcomes, the treatment continues. If the patient deteriorates, either the treatment is withdrawn (shifting the goal to palliation) or 
further treatment renegotiated if significant clinical uncertainty remains ${ }^{10}$. Trialling treatment on ICU can therefore be informative for intensivists and comforting for patients' families. Such Time Limited Trials (TLTs) are advocated in UK ICU guidelines, particularly for those patients with a high risk of dying and benefits of treatment are uncertain ${ }^{11}$, acting as a "third option" to unrestricted treatment and palliation ${ }^{12}$. TLTs are reported to maximise the chances of recovery for patients ${ }^{11}$, and prevent treatment prolongation by encouraging timely decisions, which give families the opportunity to emotionally adjust. However, TLTs have been infrequently implemented by intensivists ${ }^{13}$, being offered in only $15 \%$ of family conferences in America, and no figures available for UK hospitals ${ }^{12}$. ICU staff may therefore lack familiarity with, and experience of, TLTs.

Initiating a TLT may mark the transition from intervention to end-of-life care, which is known to be the stage most likely to lead to conflict, both within the ICU team and with the family ${ }^{14}$. There is the potential for disagreement over the length of the trial, and families requesting more time ${ }^{15}$. Furthermore, providing a trial of treatment means it will have to be withdrawn at some point if unsuccessful. Withdrawing and withholding treatment are perceived as ethically equivalent by Bioethicists and within professional guidelines; however, a minority of ICU staff do not consider them ethically equivalent and instead find treatment withdrawal more difficult in practice ${ }^{16-20}$. ICU staff have been accused of "withdrawal aversion", which may lead to harmful treatment prolongation ${ }^{21}$. This "withdrawal aversion" and resulting conflict within the ICU team or with families may explain why TLTs have had poor uptake in the UK. Palliative care was previously seen as the antithesis to intensive care by intensivists, 
but the importance of high-quality palliative care and communication on ICU have been increasingly recognised ${ }^{22-25}$.

TLTs have been proposed as a way of promoting appropriate treatment withdrawal through communication and early end-of-life discussions ${ }^{11}$, whilst giving the patient the greatest chance of recovery and reassurance to the family that all options have been exhausted ${ }^{17}$. Time, both in its presence through building relationships and in its absence by preventing treatment prolongation, seems to be significant when delivering and receiving healthcare in ICU. It is crucial therefore that we develop an understanding around how time features in ICU decision-making and how it can be used to promote positive end of life care, to gain insight into the potential utility of TLTs and how they may operate in practice. In this paper, we will explore the role of time on ICU and contribute to on-going wider debates surrounding TLTs from a UK perspective.

\section{Methods}

\section{Study design}

Qualitative interviewing was chosen to achieve greater depth in participants' responses ${ }^{26}$. A theoretical sampling technique was used to identify potential participants from two ICUs within a National Health Service Trust in North West England. These units admit general medical and surgical patients, with a capacity of eight patients on each unit. Ethical approval was granted by the Faculty of Health and Medicine Research Ethics Committee at Lancaster University, and research governance gained from the Trust's Research and Development department. 


\section{Participants/Sample}

Participants were recruitment via email and by displaying poster advertisements on the units. Participation was voluntary. Eighteen participants (nine doctors and nine nurses) with varying levels of experience in withdrawing or withholding care on ICU were recruited. All participants were given information regarding the purpose of the study and completed consent forms prior to their interview.

Data collection

In-depth, one-to-one semi-structured interviews were conducted over a three-week period in January to February 2014 using an interview guide (AW). The interviews were actively constructed narratives, where participant and interviewer are seen as equal partners in creating the participant's perspective ${ }^{27}$. Participants were given the option to terminate the interview at their convenience. Interviews lasted between 25 and 90 minutes and were audio recorded with permission.

\section{Data analysis}

An external transcriber, who had signed a confidentiality agreement, transcribed and anonymised the interview recordings verbatim. Codes were used to differentiate participants, based on their site (Site [S] 1 or 2), role (Doctor [D] or Nurse [N] and numbered consecutively at that site [1-9]) and seniority (Clinical Nurse Lead [CNL] or Staff Nurse [SN] and Consultant [C] or Junior [J] doctor). The transcripts were thematically analysed using Nvivo software, and higher themes can be identified with subsequent analyses over time ${ }^{28}$. Coding was then 
conducted in three stages (initial, axial and selective) and was informed by a review of the literature $(B L)^{29}$. Thematic coding was discussed with project supervisors (RM, LM).

\section{Results}

\section{Managing uncertainty through time}

Participants discussed the uncertainty that can exist when deciding whether treatment will be futile before it is started. Participants suggested that the source of the uncertainty stemmed from the reversibility of the patient's disease process and the patient's response to the treatment. For example, one participant queried staff's ability to predict patients' responses to any treatment provided, "Are you that good to say what is reversible and what is not?" (S1-D2-C). Such uncertainty was presented by participants as exacerbated by the urgency surrounding their decision-making, and they portrayed themselves as working with inadequate information as the following two quotes illustrate,

"You start something and then you can see over time whether that's going to improve or not work. Sometimes you don't know unless you try and give a period of time. Because sometimes that time is important" (S2-D2-J)

"All of a sudden the pathology has hit the patient very quickly, but it also hits the family very quickly and they don't really think very clearly. So, making decisions in the heat of the moment like that, I would be more inclined to treat and ask questions. It just depends how quickly the story unfolds" (S2-D3-C)

In effect, participants justified their preference for starting treatment and waiting for an unspecified period to see if the patient responded, rather than early palliation. Comparing 
the patient biochemically and clinically to his or her earlier self enabled intensivists to assess the clinical futility of treatments and avoid inappropriately prolonging dying. Time was therefore used as a tool by intensivists when judging the reversibility of illness and futility of treatment - "you can buy yourself some time to make a more informed decision" (S2-D3-C) suggesting it is not treatment alone, but its symbiotic relationship with time, that is required to navigate the uncertainty surrounding decision-making on ICU. Indeed, some participants perceived no difference between TLTs and their practice, despite the lack of formal time limits when trialling treatments.

\section{Constructing relationships through time}

Time was depicted as helping intensivists to understand their patient, the patient's family and the disease process. The strength of the bonds between ICU staff, patients and their families were depicted as developing because of the extensive time spent together on ICU. This was most frequently discussed by nurses who claimed resulted from the intensity of ICU nursing,

"Especially as we're with the patients a good 12-hour shift and you have that sort of rapport... with also the family and patient" (S2-N8-SN)

However, the relationships formed over time contributed to staff's reluctance to shift from active treatment to palliation. TLTs were therefore presented as disrupting the bond, particularly for less experienced staff, as they would otherwise find themselves "facing even more decisions about withdrawing and withholding care" (S2-D5-J).

Time as a gift 
Participants described the strong emotional reactions from family members that arose when faced with a loved one becoming ill unexpectedly and being admitted to the ICU. During the interviews, time was discussed as something that could be given to families to "take on-board what's happened" (S2-D3-C), to "work through their own thoughts and grief" (S2-N3-SN) or simply to "get the right family members in" (S2-D1-J). In these instances, participants portrayed time as a gift that demonstrated their compassion towards families and patients, which was reinforced by the implied urgency surrounding patients' ill health. The depiction of time as a compassionate gift towards families therefore portrayed staff positively.

However, the idea of time as a gift also implied that ICU staff held power as they controlled whether patients received the gift of time on ICU, as highlighted in the following two quotes,

"I don't think we should just carte blanche say everybody who gets sick should come to ITU for four days to see if we can resurrect them" (S2-D3-C)

"...it's the ones where they have the potential that they could survive, it might be quite a small potential but it's there... that you can give the chance" (S2-D4-J)

It is implied in these quotes that not every patient was deemed worthy of the gift of time, and it was the futility of treatment that could determine whether a patient was granted time on ICU. During these discussions, the gift of time was presented as something that was capped and constrained, there is "obviously a limit to the time" (S2-N3-SN), rather than time being readily available and endless as it may seem to families on admission. Participants presented time as a gift for patients, which suggested time was a valuable and limited resource to be utilised when making treatment decisions on ICU. 
Furthermore, the presentation of time as a limited gift was also apparent when participants discussed the decisions ICU staff had to make when patients were referred to ICU for admission. Intensivists claimed that families and hospital doctors had unrealistic expectations, which stemmed from their perception of time on ICU as a readily available resource. Intensivists described hospital doctors as overestimating the capacity of ICU when they referred "a lot of patients" (S1-D3-C) to ICU and families were considered unreasonable in their treatment expectations,

"the family wants everything...We are NHS and we should be realistic with what we can offer and what we can get" (S1-D2-C)

Through their depictions of time on ICU as a limited gift, participants justified their decisions to not accept every patient referred to ICU, or not to provide every available treatment to every patient. In essence, it warranted ICU staff's decision-making, and presented their rationing of care as unavoidable.

\section{Time as a form of communication}

Participants discussed the disagreements between ICU staff and a patient's family, which were most frequently surrounding the withdrawal of treatment. In these instances, participants constructed time as helping to build the evidence-base to defend staff's decision to withdraw treatment. For one participant, time was "evidence to show them [the family] that you've done what you can but it's not worked" (S2-N3-SN). Staff were portrayed as exhausting all available treatment options, and their decision as reasonable. The 'evidence' 
acquired through time was drawn upon to facilitate communicating a challenging message to the patient's family.

Participants acknowledged that "the ethos of the unit seems to be broadly similar" (S2-D6-C) and intensivists are willing to openly discuss complex cases with their colleagues. Yet, the use of time to communicate messages to families was threatened by the lack of consensus between staff over the duration needed to wait for a patient to respond to treatment. Participants proposed one to 10 days as an appropriate timeframe for patients to respond to treatment. The lack of agreement over time given led to frustration within teams, as some intensivists were accused of over-treatment and avoiding withdrawal of futile treatment for those patients with an inevitably poor prognosis,

"There are times when I felt it [treatment withholding or withdrawal] should have happened earlier...don't prolong nature, don't continue to do things that we shouldn't be doing...sometimes nurses can reach decisions a lot earlier than the doctors" (S2-N9CNL)

The inconsistency within the ICU team over the length of time to allow for a treatment to work highlighted how time significantly influenced the ability to communicate consistent messages to the family. This was most frequently discussed when participants referred to patients whose condition had stabilised over time. The decision lacked the same sense of urgency as the initial decisions the family had witnessed in life-threatening circumstances, as one participant explained when he discussed how time "seem to snowball and it becomes another day and another day", which "gives different messages to the family" (S2-D4-J). 


\section{Discussion}

In this paper, we have explored the multiple and varied roles of time on ICU. Our focus on ICU decision-making has highlighted that in addition to staff relying on each other and their experience to manage uncertainty ${ }^{4-6}$, time can also aid staff in their decision-making. Our findings have shown how ICU staff use time to construct certainty when facing decisions relating to patient prognosis or the reversibility of a disease. Moreover, the focus on ICU decision-making illustrated the importance placed by ICU staff on providing high quality end of life care for their patients ${ }^{22-24}$. In particular, staff utilised time to promote communication between staff and families, reassure families that appropriate options have been exhausted ${ }^{17}$, and prompt early end of life discussions ${ }^{11}$.

Through our study, we have gained insight into the potential utility of TLTs and how they may operate in practice in the UK. Our findings demonstrated the recognition from ICU staff that timely treatment decisions and discussions with patients and their families can support them emotionally. Staff portrayed themselves as having compassion towards patients and their families when they described giving time on ICU to benefit families emotionally. Our findings suggest that ICU staff are therefore supportive of TLTs because they have the potential to maximise the chances of recovery for patients ${ }^{11}$, and give the family a chance to emotionally adjust if the decision is made for end of life care to be initiated. Whilst no figures exist regarding the implementation of TLTs in UK hospitals ${ }^{12}$, our findings have demonstrated that ICU staff draw on time in multiple ways, such as aiding decision-making and communication with patients and families. Arguably, our findings have highlighted that ICU staff work in ways 
that are supportive of the aims and purpose of TLTs, and therefore raise the possibility that ICU staff are familiar with, and have experience of, working with time and implementing, albeit informally, TLTs. Participants raised concerns regarding the ambiguity for families when patients' conditions remain static after providing time to trial treatment. It was unclear if this observation was a by-product of staff not formally implementing a TLT or if, given the option of treatment renegotiation at the end of a trial, this ambiguity also applies to TLTs. Arguably, the setting clear goals at trial initiation could limit the ambiguity that may exist within a $\operatorname{TLT}^{11,25}$.

From our findings, we gain a UK perspective on the wider debates surrounding TLTs. The desire to formulate an agreement between staff and patients and their relatives was apparent in our data. Participants described their willingness to 'trial' treatments and presented themselves as keen to avoid conflict with patients' relatives ${ }^{12}$. It remains to be seen how long treatment could be provided for solely as a gift to the family, particularly if it is not directly in the patient's best interests. The relationship with patients and relatives was therefore constructed as valued by ICU staff. It is possible therefore that the poor uptake of TLTs in the UK may stem from ICU staff's wish to avoid conflict, and/or the challenges related to end of life care. In particular, the well-known difficulties defining and discussing futility of treatments with families ${ }^{1,2}$ and that initiating a TLT may mark the transition from intervention to end-oflife care $\mathrm{e}^{14,15}$. However, our findings do highlight that discussing and initiating end of life care is still problematic for ICU staff. This study's main limitation is that the participant's perspectives may not be representative of intensivists, particularly those working at large centralised units, elsewhere in the UK. 
Our focus on the role of time in ICU decision-making has confirmed that although deemed ethically equivalent, withdrawing treatment was perceived by our participants as more challenging for staff and families than withholding treatment in practice ${ }^{16-20}$. It is possible then that the future of TLTs in the UK may be dependent on ICU staff and their willingness and ability to engage, initiate and accept end of life discussions as part of their roles ${ }^{22-24}$. However, TLTs must be acceptable to patients and families too; ICU staff can support this by reaching a consensus within their team before they approach the family. Strict TLTs that follow known protocols need to be implemented in the UK to gain insight into proposed benefits in clinical practice ${ }^{11}$. Exploring patient and family perspectives towards TLTs is an essential next step for researchers on this topic. 


\section{References}

1. Wilkinson, DJC, Savulescu J. Knowing when to stop : futility in the intensive care unit. Curr Opin Anaesthesiolgyy 2011; 24: 160-165.

2. Coonan E. Medical Futility: A Contemporary Review. J Clin Ethics 2016; 27: 359-362.

3. Wilkinson D. The self-fulfilling prophecy in intensive care. Theor Med Bioeth 2009; 30: 401-410.

4. Marks RJ, Simons RS, Blizzard RA, et al. Predicting outcome in intensive therapy units-a comparison of Apache II with subjective assessments. Intensive Care Med 1991; 17: 159-163.

5. Vicente FG, Lomar FP, Mélot C, et al. Can the experienced ICU physician predict ICU length of stay and outcome better than less experienced colleagues? Intensive Care Med 2004; 30: 655-659.

6. Gopalan PD, Pershad S. Decision-making in ICU - A systematic review of factors considered important by ICU clinician decision makers with regard to ICU triage decisions. J Crit Care 2019; 50: 99-110.

7. Lighthall GK, Vazquez-Guillamet C. Understanding decision making in critical care. Clin Med Res 2015; 13: 156-168.

8. (FICM) F of ICM. Critical Capacity: a short research survey on critical care bed capacity. (FICM), Faculty of Intensive Care Medicine.

9. Welsh Government. Together for Health - A Delivery Plan for the Critically III. 2016; 16-24.

10. Quill, TE, Holloway R. Time-Limited Trials Near the End of Life. JAMA 2011; 306: 1483-1484.

11. Vink EE, Azoulay E, Caplan A, et al. Time-limited trial of intensive care treatment: an overview of current literature. Intensive Care Med 2018; 44: 1369-1377.

12. Schenker, Y, Tiver, GA, Yeon Hing, Seo, White D. Discussion of Treatment Trials in Intensive Care. J Crit Care 2013; 28: 862-869.

13. Society IC. Guidelines for the Provision of Intensive Care Services (GPICS). 2018; 167.

14. Coombs, MA, Addington-Hall, J, Long-Sutehall T. Challenges in transition from intervention to end of life care in intensive care: A qualitative study. Int J Nurs Stud 2012; 49: 519-27.

15. Bruce, CR, Liang, C, Blumethal-Barby, JS, Zimmerman, J, Downey, A, Pham, L, Theriot, L, Delgado, ED, White D. Barriers and Facilitators to Initiating and Completing TimeLimited Trials in Critical Care. Crit Care Med 2015; 43: 2535-43.

16. BMA Ethics. End-of-life decisions Views of the BMA, http://bmaopac.hosted.exlibrisgroup.com/exlibris/aleph/a23_1/apache_media/VITJE Q4TGE1SQBYYUCDDQ2T93SAHVT.pdf (2009).

17. Vincent JL. Withdrawing may be preferable to withholding. Crit Care 2005; 9: 226229.

18. Winter, Bob, Cohen S. ABC of intensive care: Withdrawal of treatment. BMJ 1999; 319: 306-308.

19. Sprung, CL, Paruk, F, Kissoon, N, Hartog, CS, Lipman, J, Du, B, Argent, A, Hodgson, RE, Guidet, B, Groeneveld, AB, Feldman C. The Durban World Congress Ethics Round Table Conference Report: I. Differences between withholding and withdrawing lifesustaining treatments. J Crit Care 2014; 29: 890-5.

20. Wilkinson, DJC, Savulescu J. A costly separation between withdrawing and withholding treatment in intensive care. Bioethics 2014; 28: 127-137. 
21. Wilkinson, D, Butcherine, E, Savulescu S. Withdrawal Aversion and the Equivalence Test. Am J Bioeth 2019; 19: 21-28.

22. Aslakson, RA, Curtis, JR, Nelson J. The Changing Role of Palliative Care in the ICU. Crit Care Med 2014; 42: 2418-2428.

23. Curtis JR, Vincent JL. Ethics and end-of-life care for adults in the intensive care unit. Lancet 2010; 376: 1347-1353.

24. Cook D, Rocker G. Dying with Dignity in the Intensive Care Unit. N Engl J Med 2014; 370: 2506-2514.

25. Chiarchiaro, J, White, DB, Ernecoff, NC, Buddadhumaruk, P, Schuster, R, Arnold R. Conflict Management Strategies in the ICU Differ Between Palliative Care Specialists and Intensivists. Crit Care Med 2016; 44: 934-942.

26. Silverman D. Qualitative research: theory, method and practice. 2nd Editio. London, 2004.

27. Holstein, JA, Gubrium J. The Active Interview. In: Qualitative Research Methods. Thousand Oaks, CA: SAGE Publications, 1995. Epub ahead of print 1995. DOI: 10.4135/9781412986120.

28. Howitt, D, Cramer D. Introduction to research methods in psychology. 2nd ed. Harlow: Pearson Education, 2008.

29. Attride-Stirling J. Thematic networks: an analytic tool for qualitative research. Qual Res 2001; 1: 385-405. 